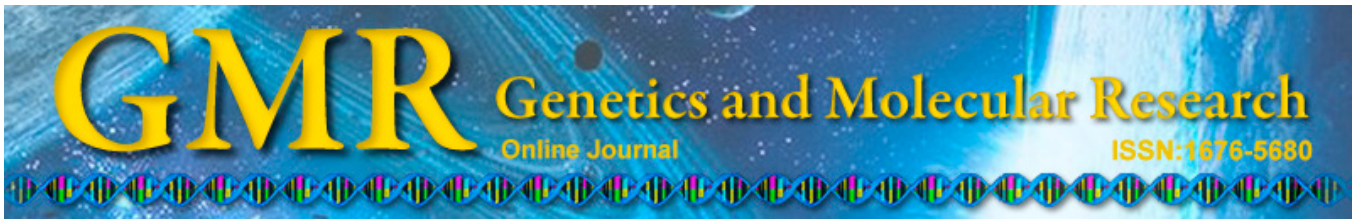

\title{
Effect of Ginkgo biloba extract on apoptosis of brain tissues in rats with acute cerebral infarction and related gene expression
}

\author{
C. Wu*, X. Zhao*, X. Zhang, S. Liu, H. Zhao and Y. Chen \\ Department of Neurology, Henan University Huaihe Hospital, Kaifeng, China \\ *These authors contributed equally to this study. \\ Corresponding author: Y. Chen \\ E-mail: yongchencn@126.com
}

Genet. Mol. Res. 14 (2): 6387-6394 (2015)

Received October 24, 2014

Accepted February 10, 2015

Published June 11, 2015

DOI http://dx.doi.org/10.4238/2015.June.11.14

\begin{abstract}
We investigated the effect of Ginkgo biloba extract on apoptosis of brain tissues in rats with acute cerebral infarction and apoptosis-related gene expression. Rat models of acute cerebral infarction were constructed using the suture method, and randomly divided into the control group, model, and treatment groups. In the treatment group, $4 \mu \mathrm{g} / \mathrm{kg} \mathrm{G}$. biloba extract was intravenously injected into the rat tail vein. Phosphate-buffered saline solution was injected in the model group. Seventy-two hours after treatment, rats were euthanized, and brain tissues were removed to analyze the changes in caspase-3, B-cell lymphoma 2 (Bcl-2), and Bcl-2-associated X protein (Bax) mRNA and protein levels, and variation in brain tissue cells' apoptosis indices was measured. Compared with the control group, the model and treatment groups showed significantly upregulated caspase-3, Bcl-2, and Bax mRNA and protein levels in brain tissues, but remarkably downregulated Bcl-2 mRNA and protein levels $(\mathrm{P}<$ $0.05)$. After treatment, in treatment group brain tissues, caspase- 3 and Bax mRNA and protein levels were significantly lower than those in the model group, while Bcl-2 mRNA and protein levels were higher than
\end{abstract}


that in the model group $(\mathrm{P}<0.05)$. The model and treatment groups showed increased cell apoptosis indices of brain tissues compared to the control group; after treatment, the apoptosis index in the treatment group was significantly downregulated compared with that in the model group $(\mathrm{P}<0.05)$. In conclusion, G. biloba extract significantly reduced apoptosis in rat brain tissue cells with acute cerebral infarction and thus protected brain tissues.

Key words: Acute cerebral infarction; Apoptosis index; Caspase-3; B-cell lymphoma 2

\section{INTRODUCTION}

Acute cerebral infarction has a high incidence and death rate; this cardiovascular and cerebrovascular disease severely threatens human health (Arboix and Alio, 2012; Mokin et al., 2014). Acute cerebral infarction increases blood viscosity and causes thrombus formation, resulting in vascular occlusion in the brain, followed by ischemia and hypoxia of brain tissues. Blood racanalization activates an ischemia reperfusion injury cascade, which releases a large amount of free radicals to act on brain cell membranes. As a result, brain cells are oxidized, and in severe cases, this cell damage is irreversible, causing extensive apoptosis and necrosis in brain cells (Candelario-Jalil, 2009; Hsieh and Chiou, 2014). Accordingly, the treatment of acute cerebral infarction is based on the effective removal of radicals from brain tissues and disconnecting the cascade damage caused by radicals. In recent years, numerous drugs have been used to treat acute cerebral infarction by removing radicals. A previous study found that some natural plant components can significantly scavenge free radicals and are antioxidant (Wang et al., 2005). Ginkgo biloba extract is purified from G. biloba, which is rich in biologically active ingredients, including the ginkgo flavonoids isoginkgetin, kaempferol, quercetin, ginkgetin, ginkgolide, and bilobalide. These chemicals can scavenge free radicals and resist lipid peroxidation (Stefanovits-Bányai et al., 2006; Kobus-Cisowska et al., 2014). Radicals and lipid peroxidation are the major causes of brain cell apoptosis and necrosis. However, few studies have examined the effects of G. biloba on brain cellular apoptosis (Smith et al., 1996; Kaur et al., 2012). The development of molecular biology and animal models has enabled analysis of the biological function of drugs on the animal scale. Therefore, we developed an acute cerebral infarction model to analyze the effect of $G$. biloba extract on brain tissue apoptosis of acute cerebral infarction rats and apoptosis-related gene expression to provide theoretical basis for the treatment of acute cerebral infarction using G. biloba.

\section{MATERIAL AND METHODS}

\section{Acute cerebral infarction model establishment and treatment}

Sprague-Dawley rats weighing 180-200 g were purchased from HFK Bioscience Co. Ltd. (Beijing, China). An arterial infarction model in the rat brains was established using the method described by Longa et al. (1989). Two hours after surgery, rats were apathetic, showed ipsilateral Horner's syndrome, contralateral forelimb sagging, adduction, and internal rotation, and were automatically circling to ipsilateral sides, demonstrating that the model was 
successively established. If a rat did not show these manifestations, the rat was excluded from further analysis. Three groups were established: sham (control group), model, and treatment groups, with 15 rats in each group. In the sham group, rats' blood vessels were separated without suture. In the model and treatment groups, the previous model establishment procedure was used. One hour after model establishment, G. biloba extract was injected intravenously into the tail vein, and $4 \mathrm{~h}$ later, $4 \mu \mathrm{g} / \mathrm{kg}$ G. biloba extract was immediately injected. In the model and control groups, an equal volume of phosphate-buffered saline (PBS) solution was intravenously injected into the rat tail vein. This study was performed in strict accordance with the recommendations in the Guide for the Care and Use of Laboratory Animals of the National Institutes of Health (Bethesda, MD, USA), Eighth Edition, 2010. The animal use protocol was reviewed and approved by the Institutional Animal Care and Use Committee of Henan University Huaihe Hospital.

\section{Reverse transcription-polymerase chain reaction (RT-PCR)}

Seventy-two hours later, rats in the 3 groups were euthanized, after which $0.1 \mathrm{~g}$ brain tissues was placed in $1 \mathrm{~mL}$ Trizol solution (Invitrogen, Carlsbad, CA, USA). After homogenization, $200 \mu \mathrm{L}$ chloroform was added to the sample. The samples were mixed by shaking, the solution was placed on ice for $15 \mathrm{~min}$, and then centrifuged at $15,000 \mathrm{~g}$ for $15 \mathrm{~min}$. The supernatant was then mixed with $500 \mu \mathrm{L}$ isopropanol, placed on ice for $15 \mathrm{~min}$, and centrifuged at $15,000 \mathrm{~g}$ for $10 \mathrm{~min}$ to pellet the RNA. The pellet was washed twice with pre-cooled $75 \%$ ethanol, and the precipitate was dissolved in double-distilled diethylpyrocarbonate-treated water. The sample concentration was determined, and RNA was transcribed into cDNA using a reverse transcription kit (TaKaRa, Dalian, China).

Based on the caspase-3, B-cell lymphoma2(Bcl-2), andBcl-2-associated protein X(Bax) mRNA sequences provided by GeneBank, the following primers were designed: caspase-3-F: 5'-GGTATTGAGACAGACAGTGG-3'; caspase-3: 5'-CATGGGATCTGTTTCTTTGC-3'. $\beta$-Actin-F: 5'-GCGGGAAATCGTGCGTGAC-3', $\beta$-actin-R: CGTCATACTCCTGC TTGCTG-3'.

First, primer specificities and annealing temperatures were optimized. Second, the following reaction system was prepared: $10 \mu \mathrm{L} 2 \mathrm{X}$ SYBR Green general qPCR Master Mix (Roche, Basel, Switzerland), $0.6 \mu \mathrm{L} 10 \mu \mathrm{M}$ upstream/downstream primers, $8.8 \mu \mathrm{L}$ cDNA diluted at $1: 100$, in a $20-\mu \mathrm{L}$ total reaction volume. Third, the reaction mixture was centrifuged at $1800 \mathrm{~g}$ and threw to pipe bottom. PCR was conducted using the following reaction conditions: pre-denaturation: $95^{\circ} \mathrm{C}, 30 \mathrm{~s}$; denaturation: $95^{\circ} \mathrm{C}, 3 \mathrm{~s}$; and annealing extension: $60^{\circ} \mathrm{C}, 30 \mathrm{~s}$. A dissociation curve was constructed, and the data were read directly from the real-time fluorescence quantitative PCR device (Bio-Rad, Hercules, CA, USA).

\section{Western blotting}

One gram of rat brain tissue from each treatment group was placed in a $1.5-\mathrm{mL}$ centrifuge tube containing $200 \mu \mathrm{L}$ tissue lysates, sonicated to homogenize the sample, placed on ice for $30 \mathrm{~min}$ for complete lysis, and centrifuged at $15,000 \mathrm{~g}$ for $15 \mathrm{~min}$. The supernatant was removed to detect protein concentration, and $4 \mathrm{X}$ loading buffer was added; after boiling for $30 \mathrm{~min}$, centrifugation, and loading into the gel, sodium dodecyl sulfate-polyacrylamide gel electrophoresis was performed. The proteins in the gel were transferred to a polyvinylidene fluoride membrane and blocked with 5\% skim milk. Caspase-3 primary antibody (Santa Cruz 
Biotechnology, Santa Cruz, CA, USA) was incubated with the membrane overnight, and then the membrane was washed thrice with PBS containing Tween 20 (PBST), incubated for $1 \mathrm{~h}$ with goat anti-mouse secondary antibody coupled to horseradish peroxidase (ZSGB-BIO Co. Ltd., Beijing, China), and washed thrice with PBST. Luminescent liquid was added for color development and to acquire images. Using $\beta$-actin as an internal reference, the gray scale of the targeted protein band was analyzed using the grayscale calculation software, and the relative expression of the targeted protein was calculated.

\section{Terminal deoxynucleotidyl transferase dUTP nick end labeling (TUNEL) staining analysis}

The brain tissues of rats in all groups were rapidly removed after euthanization and optimal cutting temperature compound-embedded, and cut into 7- $\mu \mathrm{m}$-thick slices with Leica freezing microtome (Leica Biosystems, Buffalo Grove, IL, USA), and the slices were placed on slides. The slides were soaked in 4\% paraformaldehyde solution (dissolved in PBS) and incubated at room temperature for $15 \mathrm{~min}$. Excess liquid was gently removed, and the liquid around the samples on slides was carefully dried using filter paper. The slides were soaked in PBS solution and incubated at room temperature for $15 \mathrm{~min}$. Excess liquid was gently removed, and the liquid around the samples on slides was carefully dried using filter paper. Next, $2 \mathrm{mg} / \mathrm{mL}$ proteinase K solution was diluted with PBS to 1:100 to obtain a final concentration of $20 \mu \mathrm{g} / \mathrm{mL}$. Each sample was mixed with $100 \mu \mathrm{L}$ proteinase $\mathrm{K}$ solution, which was incubated at room temperature for $15 \mathrm{~min}$. The samples were washed 2-3 times by soaking in a beaker containing PBS solution. Excess liquid was gently removed, and the liquid around the samples on slides was carefully dried using filter paper. The treated samples were placed in a humidified box; $5 \mathrm{X}$ equilibration buffer was diluted with deionized water at 1:5. Next, $100 \mu \mathrm{L}$ $1 \mathrm{X}$ equilibration buffer was dripped to cover the samples, and the samples were incubated for 10-30 min. Around the equilibration area, most of the $100 \mu \mathrm{L}$ equilibration buffer was dried using absorbent paper, and $50 \mu \mathrm{L} \mathrm{TdT} \mathrm{incubation} \mathrm{buffer} \mathrm{was} \mathrm{added} \mathrm{onto} \mathrm{the} \mathrm{cells} \mathrm{over} \mathrm{a} 5-\mathrm{cm}^{2}$ area. The cells on the plastic slides were covered to ensure that the reagent was evenly distributed. Water-soaked paper towels were placed in the bottom of the humidified box. The slides were placed into the humidified box and incubated at $37^{\circ} \mathrm{C}$ for $60 \mathrm{~min}$. The box was wrapped with aluminized paper to protect from light. The plastic slides were removed, and the slices were placed into PBS solution for 5 min and incubated at room temperature. Excess liquid was gently removed, and fresh PBS solution was used for incubation for an additional 5 min at room temperature. Filter paper was used to gently remove PBS solution around and behind the samples. The samples were analyzed under a fluorescence microscope. In the apoptotic cell nucleus, we observed green fluorescence because of the existence of BrightGreen. During observation, samples were magnified by $400 \mathrm{X}$. Ten visual fields in the infarction zone and surrounding areas were randomly selected to calculate the proportion of apoptotic cells to total cells to determine the apoptotic index of brain cells.

\section{Statistical analysis}

All data were analyzed using the SPSS 17.0 software (SPSS Inc., Chicago, IL, USA); measured data are reported as means $\pm \mathrm{SD}$; analysis of variance was used to compare mea- 
sured data among groups, and the least significant difference method for pair-wise comparison between groups. $\mathrm{P}<0.05$ was considered to indicate a statistically significant difference.

\section{RESULTS}

\section{Changes in caspase-3, Bcl-2, and Bax mRNA levels in rat brain tissues}

RT-PCR was used to analyze caspase-3, Bcl-2, and Bax mRNA levels in brain tissues of rats with acute cerebral infarction treated with different methods. Compared with the control group, the model and treatment groups showed significantly increased caspase- 3 and Bax mRNA levels in brain tissues $(\mathrm{P}<0.05)$, but clearly decreased Bcl-2 mRNA levels $(\mathrm{P}<0.05)$. Compared with the model group, the treatment group showed significantly decreased caspase-3 and Bax mRNA levels and remarkably increased Bcl-2 mRNA levels $(\mathrm{P}<0.05)$ (Figure 1).

A

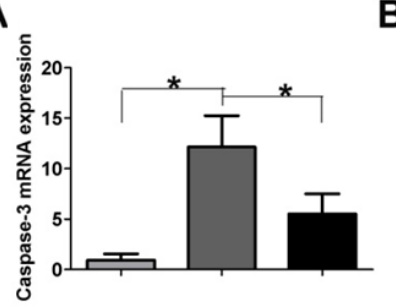

B

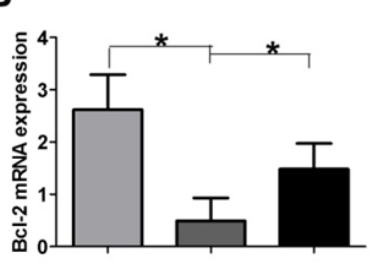

C

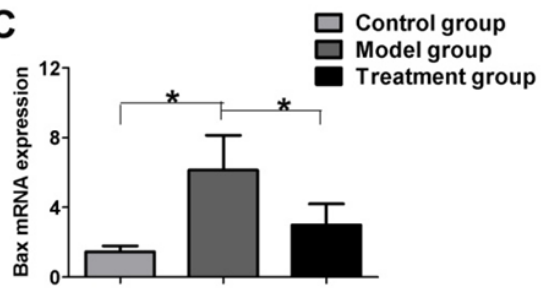

Figure 1. Caspase-3, Bcl-2 and Bax mRNA in three groups. A. Caspase-3 mRNA in control, model and treatment groups. B. Bcl-2 mRNA in control, model and treatment groups. C. Bax mRNA in control, model and treatment groups.

\section{Changes in caspase-3, Bcl-2, and Bax protein levels in brain tissues}

Changes in caspase-3, Bcl-2, and Bax protein levels in brain tissues of rats with acute cerebral infarction were examined in this study. As shown in Figure 2, in rats with acute cerebral infarction, caspase- 3 and Bax protein expression levels increased, while these levels were decreased in rat brain tissues treated with G. biloba extract. Bcl-2 protein expression was downregulated in brain tissues of rats with acute cerebral infarction, but upregulated after treatment with G. biloba extract. Quantitative analysis of caspase-3, Bcl-2, and Bax protein levels indicated that caspase- 3 and Bax protein expression in the model and treatment groups was significantly increased compared with those in the control group, but they were clearly lower in the treatment group than that in the model group $(\mathrm{P}<0.05)$. The expression of Bcl-2 protein in the brain tissues of the model and treatment groups significantly decreased compared to that in the control group, but significantly increased after treatment in the treatment group compared to that in the model group $(\mathrm{P}<0.05)$.

\section{Changes in cell apoptotic levels in brain tissues of the 3 groups}

TUNEL staining was employed in this study to analyze cell apoptosis in brain tissues following different treatments. In the model group, cell apoptosis was the most significant, and 
the apoptotic cells decreased markedly in brain tissues after treatment with G. biloba extract. Quantitative analysis of cell apoptosis in the brain tissues from rats in the 3 groups showed that compared with the control group, the model and treatment groups had significantly increased apoptosis indices in rat brain tissues $(\mathrm{P}<0.05)$, but the apoptosis index was significantly lower in the treatment group than that in the model group $(\mathrm{P}<0.05)$ (Figure 3$).$
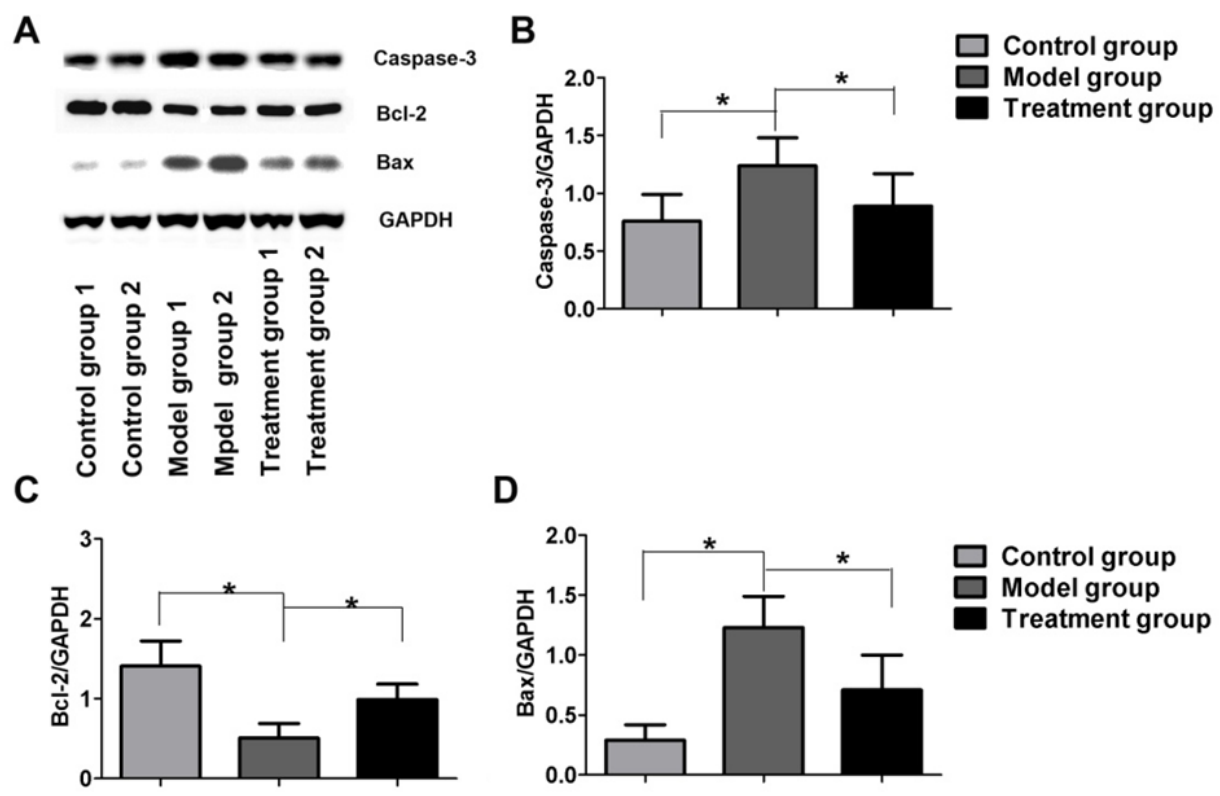

Figure 2. Caspase-3, Bcl-2 and Bax protein in three groups. A. Western blot showed Caspase-3, Bcl-2 and Bax protein in control, model and treatment groups. B. Caspase-3 protein in control, model and treatment groups; C. Bcl-2 protein in control, model and treatment groups. D. Bax protein in control, model and treatment groups.

\section{A}
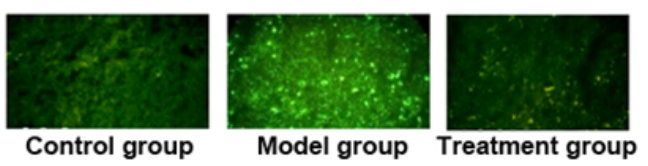

B

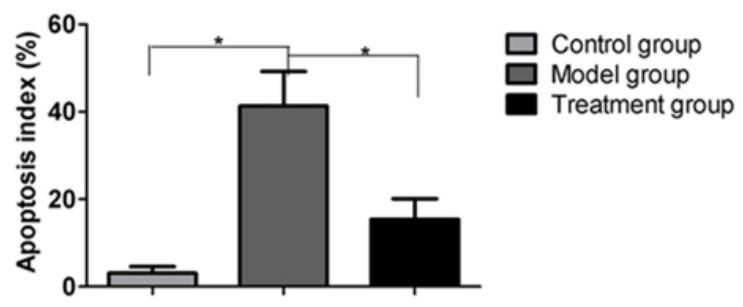

Figure 3. Apoptosis index analysis in three groups. A. TUNEL staining showed that Apoptosis index analysis in control, model and treatment groups. B. Quantitative analysis of apoptosis index in control, model and treatment groups. 


\section{DISCUSSION}

Acute cerebral infarction occurs when a blood vessel that supplies a part of the brain becomes blocked or leaks. This loss of blood supply results in oxygen deficiency and ischemia, causing infarct areas to be anaerobic and brain tissues to be depleted of energy, finally leading to brain tissue necrosis (Goldstein, 2014). Severe ischemia is typically irreversible, so acute cerebral infarction is clinically treated by reducing the damage to penumbra cells using penumbra blood perfusion. Previous studies have shown that the death of penumbra cells in brain tissues is largely mediated by cell apoptosis (Hsieh and Chiou, 2014). Cell apoptosis is genetically regulated programmed cell death, and is an automatic death process of cells regulated by genes under physiological or pathological conditions. The death of penumbra cells can be delayed by inhibiting the apoptosis of penumbra cells, which protects brain tissues (Charriaut-Marlangue, 2004; Elmore, 2007).

G. biloba extract can scavenge free radicals and resist lipid oxidation. Clinical studies have shown that $G$. biloba extract can significantly remove free radicals in patients with acute cerebral infarction and improve their hemorheological indices, protecting the nervous system (Zhuang et al., 2002; Xue and Long, 2012). However, there have been no studies examining the other biological effects of G. biloba extract. In clinical studies, we found that G. biloba improves patient nerve function and significantly decreases the infarction area, suggesting that it inhibits the apoptosis of nerve cells and brain cells. Accordingly, we verified this assumption in this study on the animal level.

In the present study, on both the RNA and protein levels, the expression of caspase-3 in rats with acute cerebral infarction was significantly decreased after injection of G. biloba extract. As a cysteine protease, caspase- 3 is the major protease participating in apoptosis. It can activate the apoptosis pathway by degrading corresponding substrates in cells, thus causing apoptosis. Thus, this protein is known as a "molecular switch" (Yu et al., 2006; Fan et al., 2014). Caspase-3 is an effector molecule shared by the external and internal apoptotic pathways. Decreased caspase-3 levels indicate that the apoptosis pathway of rat brain tissues was inhibited after treatment with G. biloba injection.

The Bcl-2 family functions as the major switch regulating the apoptosis signal pathway. Under normal conditions, the expression of Bcl-2 and Bax is stable. When Bax is overexpressed in cells, the number of Bax dimers dramatically increases, stimulating cell apoptosis. When Bcl-2 is highly expressed, Bax dimers dissociate, becoming stable complexes of Bcl-2 and Bax, prolonging cell life (Walensky, 2006; Liang et al., 2014). Therefore, the Bcl-2/Bax ratio is important in cell survival and death. Our results suggest that Bcl-2 mRNA and protein levels were markedly increased in the brain tissues of rats with acute cerebral infarction treated with $G$. biloba extract, while Bax mRNA and protein levels significantly decreased, promoting cell survival. This indicates that G. biloba extract treatment decreased Bax expression, inhibited the generation of dimers, and thus restrained apoptosis. When evaluating the overall apoptosis of brain tissues of rats, we found that the brain cell apoptosis proportion in rats treated with G. biloba extract was significantly lower than that in the model group, although still higher than that in the control group, which protects brain tissues.

In summary, G. biloba extract can significantly inhibit the apoptosis of brain tissues in acute cerebral infarction. This study provides a theoretical basis for the treatment of acute cerebral infarction with G. biloba extract. However, the mechanism of action of G. biloba extract in protecting the nervous system requires further investigation. 


\section{REFERENCES}

Arboix A and Alio J (2012). Acute cardioembolic cerebral infarction: answers to clinical questions. Curr. Cardiol. Rev. 8: 54-67.

Candelario-Jalil E (2009). Injury and repair mechanisms in ischemic stroke: considerations for the development of novel neurotherapeutics. Curr. Opin. Investig. Drugs 10: 644-654.

Charriaut-Marlangue C (2004). Apoptosis: a target for neuroprotection. Therapie 59: 185-190.

Elmore S (2007). Apoptosis: a review of programmed cell death. Toxicol. Pathol. 35: 495-516.

Fan W, Dai Y, Xu H, Zhu X, et al. (2014). Caspase-3 modulates regenerative response after stroke. Stem Cells 32: 473 486.

Goldstein LB (2014). Modern medical management of acute ischemic stroke. Methodist Debakey Cardiovasc. J. 10: 99-104.

Hsieh FI and Chiou HY (2014). Stroke: morbidity, risk factors, and care in taiwan. J. Stroke 16: 59-64.

Kaur P, Chaudhary A, Singh B and Gopichand (2012). An efficient microwave assisted extraction of phenolic compounds and antioxidant potential of Ginkgo biloba. Nat. Prod. Commun. 7: 203-206.

Kobus-Cisowska J, Flaczyk E, Rudzińska M and Kmiecik D (2014). Antioxidant properties of extracts from Ginkgo biloba leaves in meatballs. Meat Sci. 97: 174-180.

Liang K, Ye Y, Wang Y, Zhang J, et al. (2014). Formononetin mediates neuroprotection against cerebral ischemia/ reperfusion in rats via downregulation of the $\mathrm{Bax} / \mathrm{Bcl}-2$ ratio and upregulation PI3K/Akt signaling pathway. $J$. Neurol. Sci. 344: 100-104.

Longa EZ, Weinstein PR, Carlson S and Cummins R (1989). Reversible middle cerebral artery occlusion without craniectomy in rats. Stroke 20: 84-91.

Mokin M, Snyder KV, Siddiqui AH, Hopkins LN, et al. (2014). Endovascular management and treatment of acute ischemic stroke. Neurosurg. Clin. N. Am. 25: 583-592.

Smith PF, Maclennan K and Darlington CL (1996). The neuroprotective properties of the Ginkgo biloba leaf: a review of the possible relationship to platelet-activating factor (PAF). J. Ethnopharmacol. 50: 131-139.

Stefanovits-Bányai E, Szentmihályi K, Hegedus A, Koczka N, et al. (2006). Metal ion and antioxidant alterations in leaves between different sexes of Ginkgo biloba L. Life Sci. 78: 1049-1056.

Walensky LD (2006). BCL-2 in the crosshairs: tipping the balance of life and death. Cell Death Differ. 13: 1339-1350.

Wang CL, Guo F and Huang HL (2005). The research of pharmacological effects of the extracts of Ginkgo biloba leaves. Heibei Med. J. 24: 286-287.

Xue LP and Long YL (2012). Effects of Ginkgo biloba extract on platelet aggregation in patients with acute cerebral infarction. Chin. J. Integrative Med. Cardio/Cerebrovascular Dis. 10: 1188-1189.

Yu SZ, Yan L, Wang Q, An TL, et al. (2006). Effects of caspase-3 inhibitor on the neuronal apoptosis in rat cerebral cortex after ischemia-reperfusion injury. Zhonghua Bing Li Xue Za Zhi 35: 165-170.

Zhuang H, Pin S, Christen Y and Doré S (2002). Induction of heme oxygenase 1 by Ginkgo biloba in neuronal cultures and potential implications in ischemia. Cell Mol. Biol. 48: 647-653. 